\title{
TAFRO Syndrome in a Patient of South-American Descent
}

Damián Contardo ${ }^{1}$, Paola Finocchietto $^{1}$, Tatiana Uehara ${ }^{1}$, Claudia Papini $^{1}$, Natalia Deligiannis ${ }^{1}$,

Enrique Darderes ${ }^{1}$, Augusto Castroagudin ${ }^{1}$, Cecilia Cabral ${ }^{2}$, Horacio di Fonzo $^{1}$

${ }^{1}$ Department of Internal Medicine, Hospital de Clínicas “José de San Martín”, University of Buenos Aires, Argentina.

${ }^{2}$ Department of Pathology, Hospital de Clínicas “José de San Martín”, University of Buenos Aires, Argentina.

Received: 26/04/2015

Accepted: 25/06/2015

Published: $14 / 07 / 2015$

How to cite this article: Contardo D, Finocchietto P, Uehara T, Papini C, Deligiannis N, Darderes E, Castroagudin, Cabral C, di Fonzo H. TAFRO syndrome in a patient of south-american descent. EJCRIM 2015;2:doi: 10.12890/2015_000220

Conflicts of Interests: The authors declare that they have no competing interests.

Acknowledgements: PF and HdF reviewed the literature and wrote the paper. PF, DC, TH, CP, ND, AC and HdF: treated the patient and collected the data. CC performed the biopsy analysis. All authors read and approved the final manuscript.

We are grateful to Lourdes Arrubito (M.D, Ph.D) from Institute of Genetic, Immunology and Metabolism, CONICET and University of Buenos Aires for Interleukin-6 determination. The method of detection was BD Opteia Human IL-6 Elisa Set.

Written informed consent was obtained from the patient's next kin for publication of this case report and any accompanying images. A copy of the written consent is available for review by the Editor-in-Chief of this journal.

This article is licensed under a Commons Attribution Non-Commercial 4.0 License

\section{ABSTRACT}

A 31-year-old Caucasian woman of South-American descent was diagnosed with a variant of multicentric Castleman disease (MCD) that has been reported in Japan as Castleman-Kojima disease. This is a systemic inflammatory disorder known as TAFRO Syndrome which includes thrombocytopenia, polyserositis (ascites/pleural effusion), microcytic anemia, myelofibrosis, fever, renal dysfunction and organomegaly, with immunologic disorder, polyclonal hypergammaglobulinemia, and elevated levels of interleukin-6 (IL-6) and the vascular endothelial growth factor present in serum and/or effusions. Optimal therapies are not well established. The patient was treated with methylprednisolone and rituximab. Following the start of treatment, the patient has been asymptomatic for over 8 months. This is one of only a few reports of TAFRO Syndrome in a non-Japanese patient.

\section{LEARNING POINTS}

- Castleman Disease (CD) is a lymphoproliferative disorder which may exhibit a progressive, severe and life-threatening clinical course.

- TAFRO Syndrome (thrombocytopenia, anemia, systemic inflammation (ascites/pleural effusion), myelofibrosis, renal dysfunction and organomegaly) is a rare variant of CD first reported in Japan.

- The cause of the disease is unknown. Elevated levels of interleukin-6 (IL-6) and vascular endothelial growth factor in serum and/or effusions are believed to be linked to the pathogenesis.

- In this case, high doses of steroids and rituximab achieved a remission.

- This rare entity is not always confined to those of Japanese descent and can occur in Caucasian patients.

\section{KEYWORDS}

Castleman disease, TAFRO syndrome.

\section{INTRODUCTION}

Castleman Disease (CD) is an uncommon lymphoproliferative disorder diagnosed by clinical, histopathological and laboratory criteria. It was first described in 1954 by Benjamin Castleman ${ }^{[1,2]}$.

Two primary clinical presentations were characterized: the unicentric CD (UCD) confined to a single lymph node, and the multi-centric $C D(M C D)$ which compromises multiple sites ${ }^{[3]}$. Several studies have indicated that $C D$ is composed of several disease entities, including idiopathic CD and secondary CD due to human immunodeficiency type-1 (HIV) infection; autoimmune disease-associated lymphadenopathy; polyneuropathy; anasarca; organomegaly; endocrinopathy; M-proteins and skin lesions (POEMS Syndrome); and non-Hodgkin's lymphomas ${ }^{[4,5]}$. Moreover, the involvement of human herpesvirus 8 (HHV-8) infection has been demonstrated in at least $40-50 \%$ of MCD unrelated to HIV in western countries ${ }^{[6,7]}$.

Three histopathologic subtypes have been described: hyaline-vascular (HV); plasma cell; and mixed variant. Of the localized forms of CD, the HV type is found in about $85 \%$ of cases and the plasma-cell type in $15 \%[8,9,10]$.

The HV type is often asymptomatic, whereas the plasma cell type tends to exhibit a more aggressive clinical course with constitutional symptoms and laboratory abnormalities. 
A variant of MCD has been reported in Japan and labeled Castleman-Kojima disease. This is a systemic inflammatory disorder known as TAFRO Syndrome which includes thrombocytopenia, polyserositis (ascites/pleural effusion), microcytic anemia, myelofibrosis, fever, renal dysfunction and organomegaly, with immunologic disorder, polyclonal hypergammaglobulinemia, elevated levels of interleukin-6 (IL-6) and with the vascular endothelial growth factor present in serum and/or effusions ${ }^{[11,12]}$.

We describe the case of a Caucasian woman with Castleman-Kojima disease (TAFRO Syndrome), who was treated with corticosteroid and rituximab ${ }^{[13]}$.

\section{CASE REPORT}

A 31-year-old woman with no significant medical history presented with recurrent episodes of fever, night sweats and dyspnea for 2 months. Physical examination revealed fever $\left(38-38.5^{\circ} \mathrm{C}\right)$; skin pallor; enlargement of bilateral, lateral and posterior cervical and axillary lymph nodes; anasarca generalized edema; abdominal distention; tense ascites; and hepatosplenomegaly.

The patient's hemoglobin $(\mathrm{Hb})$ was $6.3 \mathrm{gr} / \mathrm{dl}$, with a low mean corpuscular volume of $76.8 \mathrm{fl}$. White blood cells were $6.9 \times 109 / \mathrm{L}$ and platelets were $69 \times 109 / \mathrm{L}$. Both creatinine (1.9 mg/dl) and urea $(150 \mathrm{mg} / \mathrm{dl})$ were elevated. Although the patient's liver function test was normal, albumin, serum iron and ferritin were elevated, suggesting a chronic inflammatory illness.

The patient's C-reactive protein was $17 \mathrm{mg} / \mathrm{dl}$, the erythroid sedimentation rate was $75 \mathrm{~mm} / \mathrm{h}$ and the direct Coombs' test resulted strongly positive with normal haptoglobin, panagglutinin immunoglobulin $\mathrm{G}$ (Ig-G) antibodies and without evidence of hemolysis.

A Mantoux test was negative as were serum hepatitis B surface antigen (HBsAg), anti- hepatitis C virus (HVC), HHV-8, anti-HIV, EpsteinBarr virus Immunoglobulin M (EBV IgM), Huddleson test, Chagas hemaglutination and antibody, venereal disease research laboratory (VDRL) quantification and toxoplasmosis antibody, cytomegalovirus (CMV) anti-lgG and CMV-polymerase chain reaction (PCR).

Anti-DNA antibody, antineutrophil cytoplasmic antibodies (ANCA), rheumatoid factor and serum complement were all normal or negative; the antinuclear antibody positive speckled pattern was 1/160; the serum total complement was normal; and beta-2 microglobulin (B2M) was $2.4 \mathrm{mg} / \mathrm{L}$. The serum IL-6 was recorded at $60 \mathrm{pg} / \mathrm{ml}(<4 \mathrm{pg} / \mathrm{ml})$. Urinalysis showed a small amount of proteinuria. An abdominal ultrasound showed hepatomegaly on the left hepatic lobe at $135 \mathrm{~mm}$ and on the right hepatic lobe at $178 \mathrm{~mm}$, with splenomegaly at $133 \times 52 \mathrm{~mm}$. Echocardiography showed normal left ventricular function with $5 \mathrm{~mm}$ of pericardial effusion, and a computed tomographic scan (Figs. 1 and 2) revealed pleural effusion, hepatosplenomegaly, ascites, adrenal glands enlarged with increased blood flow velocity in vessels without retroperitoneal lymph nodes. The ascitic and pleural fluids were both exudates.

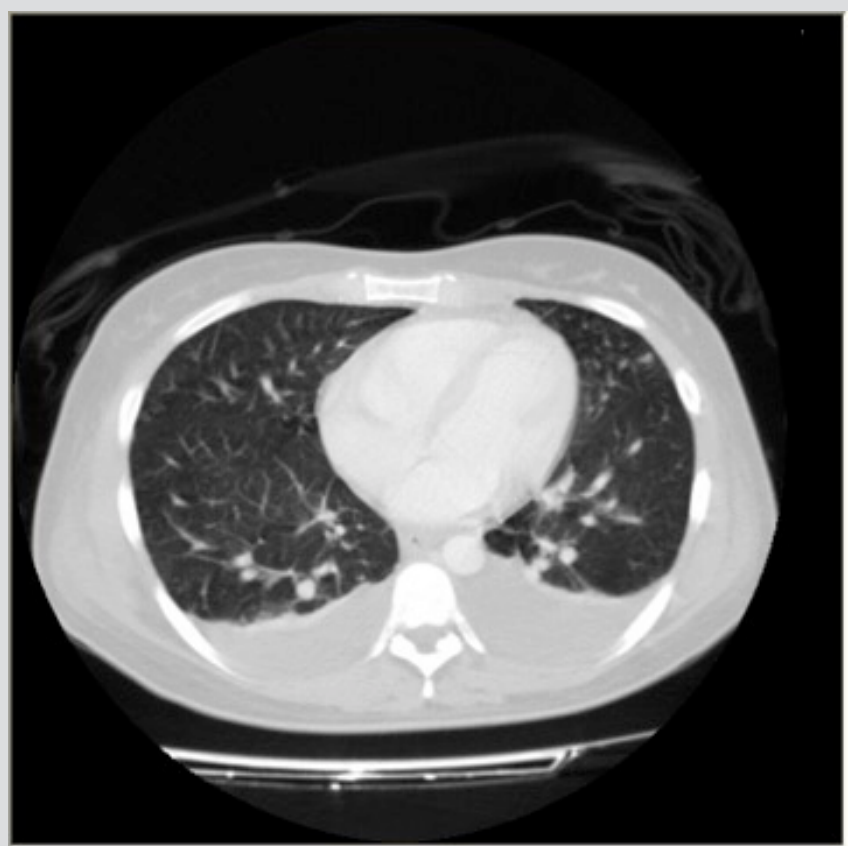

Figure 1: Contrast enhanced computed tomography scan of thorax shows pleural effusion without lymph nodes.

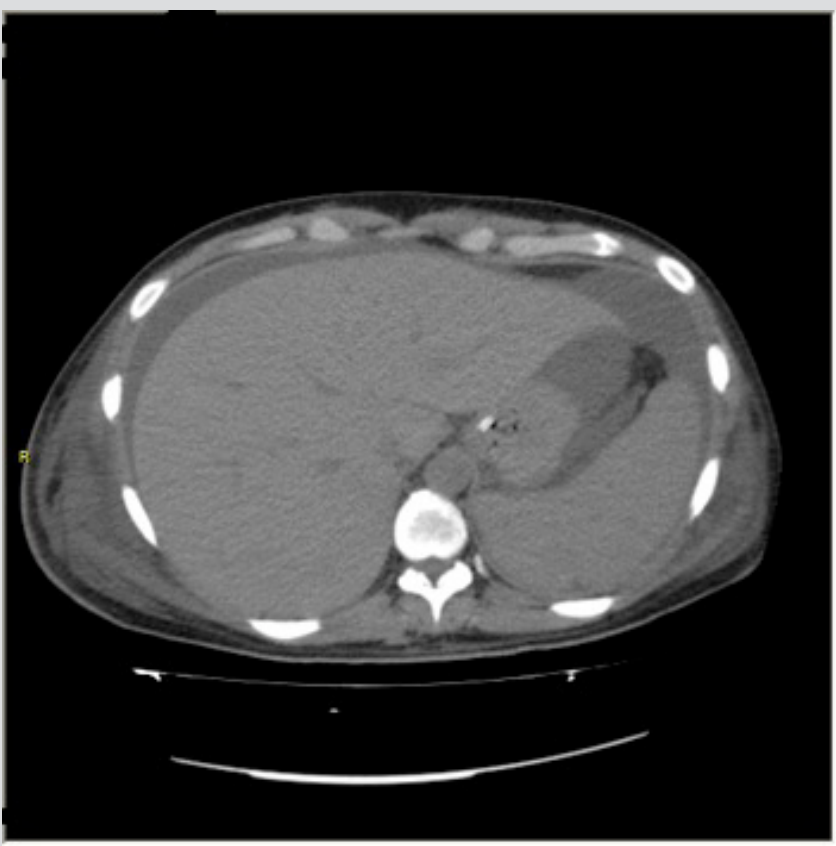

Figure 2: Contrast enhanced computed tomography scan of abdomen shows hepatosplenomegaly, ascites, adrenal glands enlarged and flow of blood vessels increased without retroperitoneal lymph nodes

A right axillary lymph node biopsy is shown in Fig. 3. The immunohistochemical inspection resulted negative for CD20, PAX5 y CD78a, Bcl-2, CD30, CD15 y Alk in follicular region and lymphocytes-T positive for CD3, CD5, CD43, CD30+ and CD79a in the interfollicular area. A bone marrow biopsy showed normal morphology and adequate marrow iron, and the reticulin stain showed grade I fibrosis. The HHV-8 marker was negative.

The patient was transfused with units of packed erythrocytes, and treated with $500 \mathrm{mg} / \mathrm{d}$ IV of methylprednisolone and $375 \mathrm{mg} / \mathrm{m}^{2}$ of rituximab, once weekly for 4 doses.

The patient has remained asymptomatic for over 8 months. 


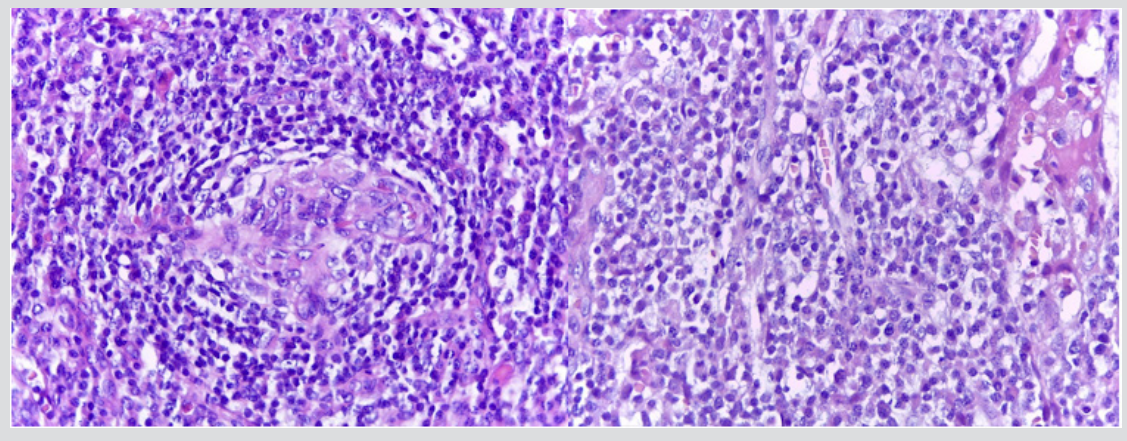

Figure 3: Lymphoid proliferation where the follicles were regressed with interfollicular region and paracortical area, expanded with lymphocytes distributed as "onionskin" fashion with follicular dendritic cells, plasma cells and variable blood vessels. H\&E 250x.

\section{DISCUSSION}

The Castleman-Kojima disease occurs in the middle aged, without any prior history of autoimmune diseases and is four 4 times more common in women than in men.

Kojima et al. reported that MCD in Japan is a hyper-IL-6 syndrome and usually occurs without either HIV or HHV8 infection. It exhibits a chronic disease course, and the authors published 7 cases in which effusions were the initial clinical presentation ${ }^{[14]}$.

The following criteria have been established for the diagnosis of TAFRO syndrome: thrombocytopenia, microcytic anemia, systemic inflammation (Ascites/pleural effusion), myelofibrosis, fever, renal dysfunction and organomegaly. Antinuclear antibody and immunologic disorder (rheumatoid factor, platelet-associated $\operatorname{lgG}$, anti-thyroid antibody) may also appear during the course of the illness ${ }^{[15]}$.

The treatment of CD is not well established. Steroids are commonly used and can achieve remission in up to $60 \%$ of cases, however recurrence rates are high. The role of IL-6 in the pathogenesis of the disease has prompted the use of the anti-receptor antibody IL-6 tocilizumab, which has been reported to induce complete remission in several cases ${ }^{[16,17]}$. The monoclonal anti-CD20 antibody rituximab has also been successfully used, either alone or in combination with chemotherapy ${ }^{[18]}$. In the present case, rituximab associated with steroids resulted in complete remission. Treatment with chemotherapy (e.g. cyclophosphamide, vincristine, doxorubicin and prednisone or dexamethasone) also has been reported to achieve high rates of remission, however it provides little chance of recurrence-free survival. Durable responses occur in only $25 \%$ of cases, but rarely remissions have been sustained for 15 years ${ }^{[16]}$. Recurrences after remission worsen the prognosis and limit the chances of survival. Further studies to determine the optimal treatment of this condition are needed.

\section{CONCLUSIONS}

Our case meets all the criteria for TAFRO Syndrome in a Caucasian patient of South-American descent. Several questions remain regarding the mechanism and etiology of this rare disease. It is still controversial whether TAFRO Syndrome should be considered as a variant of MCD or if it is a distinct entity. Clinical presentation is dominated by serositis associated with thrombocytopenia, it is not associated with $\mathrm{HHV}-8$ infection, and it has a chronic indolent clinical course.

\section{REFERENCES}

Castleman B, Towne VW. Case records of the Massachusetts General Hospital. Case 40231. NEJM 1954;23:1001-1005.

2. Castleman B, Iverson L, Menendez VP. Localized mediastinal lymphnode hyperplasia resembling thymoma. Cancer 1956;9:822-30.

Jongsma TEF, Verburg RJ, Geelhoed-Duijvestijn PHLM. Castleman's disease: A rare lymphoproliferative disorder. Eur J of Intern Med 2007;18:87-89.

Kojima M, Shimizu K, Ikota H, Ohno Y, Motoori T, Itoh H, et al. "Follicular variant" of hyaline-vascular type of Castleman's disease: histopathological and immunohistochemical study of 11 cases. J Clin Exp Hematop 2008;48:39-45.

5. Kojima M, Nakamura N, Tsukamoto N, Yokohama A, Kobayashi S, Kashimura M. Multicentric Castleman's disease representing effusion at initial clinical presentation: clinicopathological study of seven cases. Lupus 2011;20:44-50.

6. Talat N, Schulte KM. Castleman's disease: systematic analysis of 416 patients from the literature. Oncologist 2011:16:1316-24

7. Robinson D, Casper C, Vermeulen J, Reynolds M, Dispenzieri A, Payne K, et al. Clinical epidemiology and treatment patterns of patients with multicentric Castleman disease: results from two US treatment Robinson D, Casper C, Vermeulen J, Rey

8. Muzes G, Sipos F, Csomor J, Sréter L. Multicentric Castleman's Disease: A Challenging Diagnosis. Pathol Oncol Res 2013,19:345-351.

. Soumerai JD, Sohani AR, Abramson JS. Diagnosis and Management of Castleman Disease. Cancer Control 2014;21:266-278.

10. Mian H, Leber B. Mixed variant multicentric Castleman disease treated with Rituximab: case report. J Pediatr Hematol Oncol 2010;32:62

11. Kawabata H, Takai K, Kojima M, Nakamura N, Aoki S, Nakamura S, et al. Castleman-Kojima Disease (TAFRO Syndrome): A novel systemic inflammatory disease characterized by a constellation of symptoms, namely, thrombocytopenia, ascites (anasarca), microcytic anemia, myelofibrosis, renal dysfunction, and organomegaly: A Status Report and Summary of Fukushima (6 June, 2012) and Nagoya Meeting (22 September, 2012). J Clin Exp Hematop 2014;2:163-166.

12. Koduri PR, Parvez M, Kaza S, Pappu P, Anuradha S. Castleman-Kojima Disease in South Asian Adolescent. J Clin Exp Hematop 2013;53:57-61.

13. Marcelin AG, Aaron L, Mateus C, Gyan E, Gorin I, Viard JP, et al. Rituximab therapy for HIV-associated Castleman's disease. Blood 2003, 102:2786-2788

14. Kojima M, Nakamura N, Tsukamoto N Otuski Y, Shimizu Ket al. Clinical implications of Multicentric Castleman's disease among Japanese. A report of 28 cases. Int J Surg Pathol 2008:16:391-398.

15. Masaki Y, Nakajima A, Iwao H, Kurose N, Sato T, Nakamura T, et al. Japanese variant of multicentric Castleman's disease associated with serositis and thrombocytopenia-a report of two cases: is TAFRO syndrome(Castleman- Kojima disease) a distinct clinicopathological entity? I Clin Exp Hematop 2013;1:79-85.

16. Tedesco S, Postacchini L, Manfredi L, Goteri G, Luchetti MM, Festa A et al. Successful treatment of a Caucasian case of multifocal Castleman's disease with TAFRO syndrome with a pathophysiology targeted therapy - a case report. Exp Hematol Oncol 2015;4:3.

17. Robinson D, Casper C, Vermeulen J, Reynolds M, Dispenzieri A, Payne K et al. Clinical epidemiology and treatment patterns of patients with multicentric Castleman disease: results from two US treatment centres. Br J of Haematol 2014;165:39-48.

18. Zhu SH, Sun JJ, Yu YH, Han DL, Li J, Zhang Y. Clinical features and outcome of patients with HIV-negative multicentric Castleman's disease treated with combination chemotherapy: a report on 10 patients. Med Oncol 2013;30:492. 